\title{
LASER Microdissection of Metaphase CHROMOSOMES AND CHARACTERIZATION By Atomic Force Microscopy
}

\author{
Stefan Thalhammer, ${ }^{\dagger}$ Robert W. Stark, ${ }^{\dagger}$ Karin Schütze,${ }^{*}$ Johannes Wienberg, ${ }^{*}$ \\ and Wolfgang M. Heckl ${ }^{\dagger}$ \\ †University München, Institute for Crystallography, Theresienstr. 41, 80333 München, Germany; \\ ${ }^{\dagger}$ Harlaching Hospital, München, Germany; *University of Cambridge, Department of Pathology, \\ Cambridge, United Kingdom \\ (Paper JBO-093 received May 13, 1996; revised manuscript received Sep. 30, 1996; accepted for publication Oct. 15, 1996.)
}

\begin{abstract}
A new experimental setup has been constructed in which a UV laser microbeam and atomic force microscopy (AFM) have been combined on an inverted microscope in order to manipulate and visualize chromosomes with high resolution. The laser beam has been used to dissect Muntjak metaphase chromosomes and was aimed to optimize the physical size of the cuts. The capability of the AFM to visualize biological material with relative ease has been used to characterize the microdissected chromosomes. This work demonstrates that chromosome fiber material can be removed completely at the cut site using appropriate laser power. The minimum cut size achieved with a 337-nm nitrogen UV laser was between 600 and $800 \mathrm{~nm}$. The smallest distance between the cuts was around $500 \mathrm{~nm}$, corresponding to the finest probe for further biochemical use after physical translocation such as the polymerase chain reaction. Limitations on minimizing the cut size due to diffraction-limited focusing and the effects of laser ablation of biomaterial are discussed. ( $) 1997$ Society of Photo-Optical Instrumentation Engineers.
\end{abstract}

Keywords laser micromanipulation; laser dissection; atomic force microscopy (AFM); chromosomes; cytogenetics.

\section{INTRODUCTION}

Physical dissection of metaphase chromosomes is the most advanced approach for isolating DNA sequences from specific chromosome regions. ${ }^{1}$ In the past few years different techniques have been used for this purpose, including laser microdissection, ${ }^{2}$ atomic force microscopy (AFM) in combination with isolation of specific regions, ${ }^{3,4}$ and silanized glass needles, where additional cloning of defined regions of the human genome and enzymatic amplification has been demonstrated. ${ }^{5}$ Atomic force microscopy has proven to be an excellent tool for visualizing chromosomes. ${ }^{6,7}$ Its range extends from dye-labeled, highly condensed human metaphase spreads in combination with in situ hybridization (ISH) technique ${ }^{8}$ and near-field optical imaging, ${ }^{9}$ to chromatin fibers ${ }^{10}$ and down to single plasmid DNA strands. ${ }^{11}$

Here we describe the use of laser microdissection combined with AFM to visualize and analyze metaphase chromosomes which were cut by a UV laser beam. "Noncontact" micromanipulation with different laser beams as highly precise tools has been used in several areas of cellular and molecular

Address all correspondence to Wolfgang M. Heckl. E-mail: w.heckl@lrz.uni-muenchen.de biology as well as in medical applications. ${ }^{12-14}$ Chromosome cutting in vitro and in vivo has been demonstrated in several laboratories. Recently, Lengauer et al. demonstrated that even DNA strands visualized with fluorescent dyes could be precisely cut with UV laser microbeams. ${ }^{2}$ Compared with chromosome cutting with needles, the laser method is quick and easy and does not require the timeconsuming preparation of microneedles. However, the biggest advantage of the laser cutting method is its possibility for generating probes without any mechanical contact and therefore avoiding the risk of contamination. This makes it easy to further use the probes for genetic engineering with techniques such as polymerase chain reaction (PCR) amplification.

In order to evaluate the size and the quality of the laser microdissection, a high-resolution microscopic technique should be used. Electron microscopy is inconvenient for this purpose because it cannot be used in situ and requires preparation techniques, such as metal staining. Therefore we used a standalone AFM which was mounted on an inverted microscope stage in combination with a UV laser mi-

1083-3668/97/\$10.00 @ 1997 SPIE 


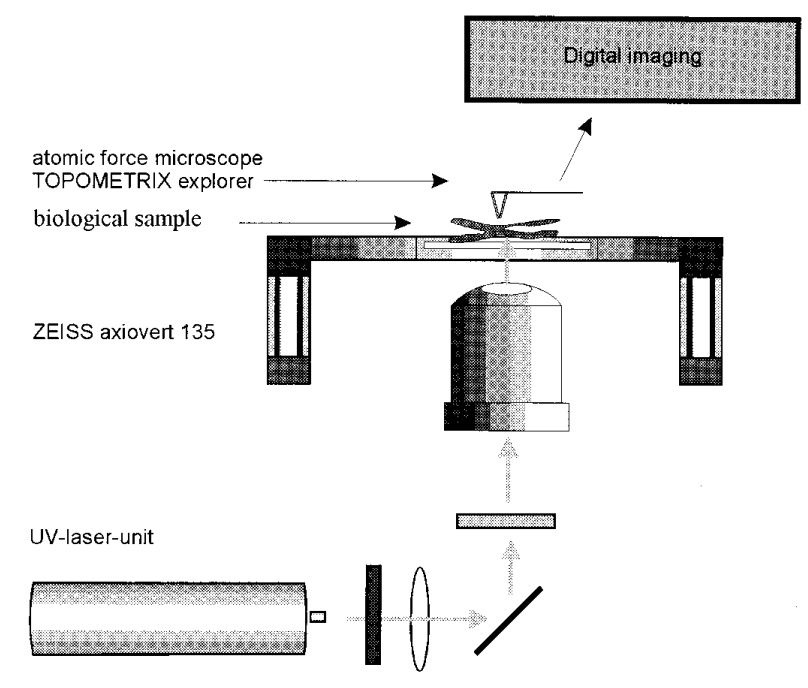

Fig. 1 Schematic of the experimental setup used to microdissect chromosomes with a laser microbeam and to analyze the cuts with an AFM.

crobeam for direct quality analysis of the micromanipulated biological material.

\section{MATERIAL AND METHODS}

\subsection{PREPARATION OF METAPHASE SPREADS}

Fibroblast cells of Muntjak muntiacus were cultivated in Dulbecco's modified Eagle's medium (DMEM) media (containing 10\% fetal calf serum (FCS), $2.5 \%$ glutamine, $1 \%$ antibiotic mixture (streptomycin-penicillin)) for $72 \mathrm{~h}$ at $37^{\circ} \mathrm{C}$ with $5 \%$ $\mathrm{CO}_{2}$ and $80 \%$ atmospheric humidity. Cells were arrested in metaphase with $0.05 \mu \mathrm{g} / \mathrm{ml}$ Colcemid 1 to $2 \mathrm{~h}$ before fixation, followed by the trypsinization step $(0.05 \%$ trypsin solution) to harvest the cells. After centrifugation, hypotonic treatment $(0.075 \mathrm{M}$ $\mathrm{KCl}, 0.4 \%$ sodium citrate) and fixation of the cells were performed according to standard protocols. Metaphases were prepared by drop fixation on cover slides specially designed for laser microdissection, air dried, dehydrated with ethanol, and stored in $70 \%$ ethanol at $4{ }^{\circ} \mathrm{C}$ until use. Before the slides were used for laser microdissection and AFM imaging, they were treated with a pepsin solution (50 $\mathrm{\mu l}$ of a $10 \%$ stock solution in $100 \mathrm{ml}$ of $0.01 \mathrm{M}$ $\mathrm{HCl}$ ) for $5 \mathrm{~min}$ at $37^{\circ} \mathrm{C}$ and then washed twice in $1 \times$ phosphate buffered saline (PBS) for $5 \mathrm{~min}$ and rinsed in water; they were dehydrated by an ethanol series (70, 90, and 100\%) and air dried.

\subsection{COMBINED LASER MICROBEAM AFM SETUP}

Figure 1 shows the experimental setup. A UV laser microbeam (P.A.L.M. GmbH Wolfratshausen) consisting of a 337-nm nitrogen laser coupled through the epi-illumination path of an inverted research microscope (ZEISS Axiovert 135) has been com-

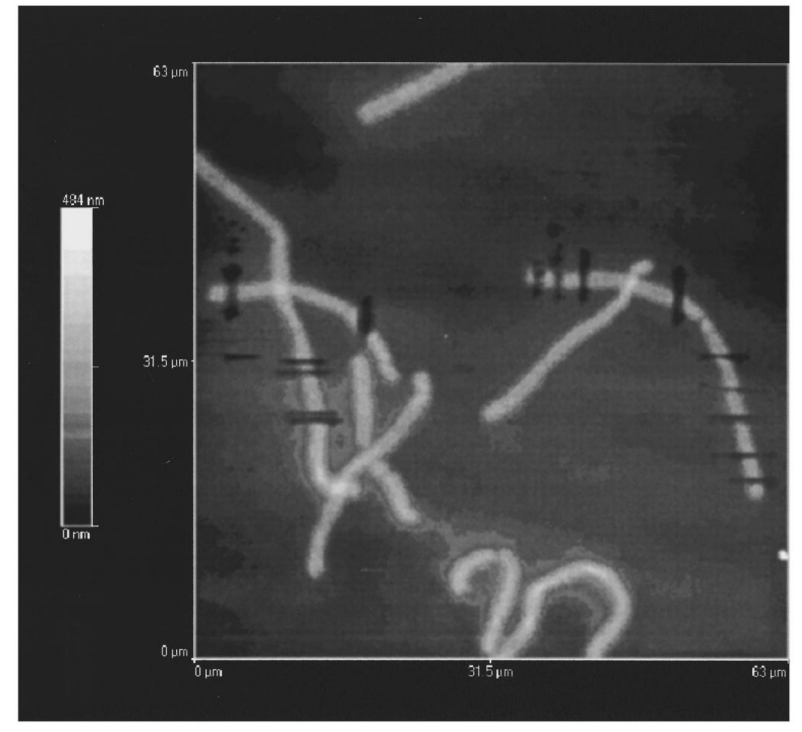

Fig. 2 AFM micrograph of a Muntjak metaphase chromosome spread in which brighter pixels correspond to regions of increased height.

bined with a stand-alone AFM (TopoMetrix Explorer). The UV laser is focused to a spot size of less than $1 \mathrm{~m} \mu^{3}$ using a high numeric aperture objective (Plan Neofluar $100 \times 1.3$ oil, $\mathrm{Ph} 3$ ). A special laser microscope interface allows minute laser focus adjustments, independent of the microscope focus. Thus, the center of the beam waist can be focused exactly on the target, yielding the smallest possible laser cuts. Maximum laser output energy is about $300 \mu \mathrm{J}$. The laser energy was continuously attenuated without beam displacement using a motorized laser attenuator. For chromosome cutting we usually used about $1 \mu \mathrm{J}$ per pulse. The laser was triggered by a pushbutton that could be used in single or repetitive pulse mode. The pulse duration was 3 ns, and the pulse repetition rate was up to 15 pulses per second. After laser cutting in air, AFM was performed in air using $\mathrm{Si}_{3} \mathrm{~N}_{4}$ cantilevers with a force constant of $0.003 \mathrm{~N} / \mathrm{m}$ in the constant force mode with an imaging force of $5 \mathrm{nN}$. The design of the stand-alone AFM allows the cantilever to scan the surface topography from above the specimen so that three different microscope techniques can be combined and used simultaneously: nanomanipulation via laser microbeam techniques, optical monitoring through the eyepiece/CCD camera of the inverted microscope, and high-resolution AFM imaging of the surface topography.

\section{RESUlTS AND DisCUSSION}

Figure 2 shows an AFM image of the topology of a Muntjak metaphase plate imaged with a standard pyramidal tip after several laser cuts have been made with 15 pulses of 3 ns duration and $1 \mu \mathrm{J}$ per pulse. The laser cut pierces through the metaphase chromosome until it reaches the underlying glass 


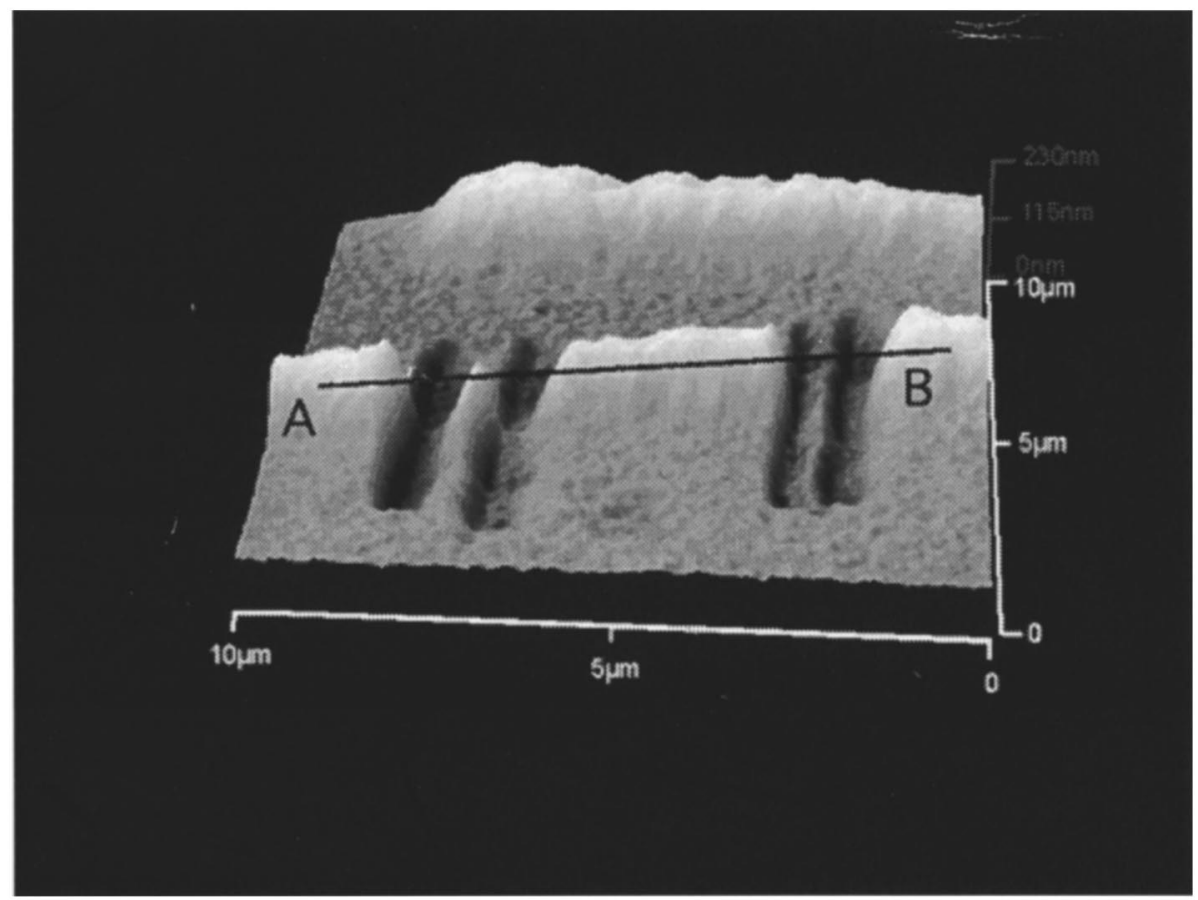

(a)

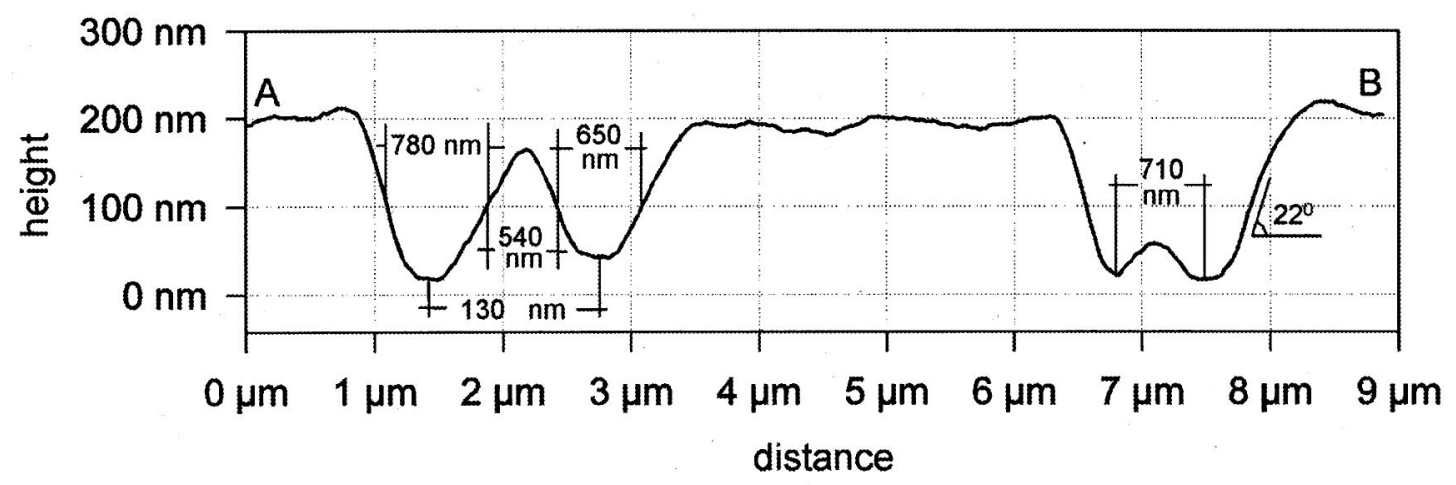

(b)

Fig. 3 (a) Closeup of two laser double cuts in a 3-D representation and (b) cross-section of the chromosome with four laser cuts along the dashed line $A B$.

substrate. Each cut has produced about the same cross-sectional depth in the chromosome and, while scanning with the AFM in constant force mode, no loose debris of biological material derived from chromosomes, proteins, or cell components could be detected. This can be explained by cold laser ablation. Contrary to a cutting technique using needles or an AFM tip, there was no contiguity of the chromosome and consequently no dragging of DNA material along the scan path, which we have observed with AFM cutting. ${ }^{15}$ In addition, the method of pulsed energy deposition in laser cutting probably leads to minute bursts of biomaterial when molecular bonds are broken during the absorption of UV light with an energy density of up to $10^{12} \mathrm{~W} / \mathrm{cm}$ at the focal point. Consequently the material is distributed within a relatively large area.

Figure 3(a) is an enlargement of a laser double cut in a 3-D representation. The details of these four cuts have been analyzed via a line profile drawn along the line at the apex of the chromosome [Figure 3(b)]. The cross-section profile reveals a fullwidth-at-half-maximum-height (FWHM) width of the laser cut of around $700 \mathrm{~nm}$ with a cutting angle of around $22 \mathrm{deg}$ with respect to the surface. The 
half-width describes the cross-section width, where the flank height is $50 \%$. Here it is assumed that in the AFM image the convolution of the pyramidal tip with the chromosome cut narrows the actual physical cut size by two times the size of the tip apex of around $10 \mathrm{~nm} .{ }^{16}$ This is not important compared with the size of the cuts. The distances between the cuts of the two pairs measure 1300 and $700 \mathrm{~nm}$, respectively. The larger cut pair leaves a 540-nm chromosome part in between with a maximum height between 100 and $170 \mathrm{~nm}$.

Compared with the undisturbed regions of the chromosome with a height of around $200 \mathrm{~nm}$, in structural terms this part is no longer fully intact. This loss of height could be due either to removal of material (then the chromosome is no longer intact) or to a melting of the material with a subsequent condensation, which could leave the DNA in place. In this case, the region would still be genetically intact and could possibly be used for further biological treatment. The smaller cut shows that the height of the chromosome material in between is substantially reduced compared with the normal apparent height of the dehydrated intact chromosome of around $200 \mathrm{~nm}$. For the effect of hydration on the volume of human chromosomes, see Ref. 17. Therefore we conclude that the two cuts have been made too close together, leaving no intact chromosome probes that can be used for further applications.

There are two possible reasons for the limit on the cutting size. First, the laser can only theoretically be focused to a diffraction-limited spot size of around $200 \mathrm{~nm}$. In practice lens imperfections and the loss of beam quality caused by optics that are not optimally corrected within the microscope lead to widening of the laser beam. Also, the adjustment of the focal point is critical. Further reduction of the point spread function through a confocal geometry or special setups such as the confocal theta construction with improved axial resolution by a factor of 3.5 (Ref. 18) are essential. Another principal reason lies in the mechanism of the radiation transfer during cold laser ablation of the biomaterial. As described above, during the minute time of the laser shot a gaseous burst of biomaterial explodes on the surface of the chromosomes, tearing material from the side and thus broadening the cut. This effect should destroy the genetic integrity of the affected regions. How far this region reaches into the intact part of the chromosome cut cannot be determined by surface profile measurements, but only by determining biochemical activity. This interpretation of "franzzling" at the laser cut side is also supported by the fact that the cut profile angle of around 25 deg is very flat compared with the expected $50 \mathrm{deg}$ for a steep laser cut with the convolution of a pyramidal AFM tip profile.

\section{SUMMARY}

In a combined setup, AFM is perfectly suitable for analyzing laser dissection of chromosomes. To achieve laser cuts of around $700 \mathrm{~nm}$ within the chromosomes, a minute laser focus and energy adjustment are necessary. The limit in the probe size to be generated by the laser cutting technique is around $500 \mathrm{~nm}$, which corresponds to several megabases in the case of a highly condensed metaphase chromosome. This is due to the absorption behavior of the target as well as the limits in focusing the laser beam. The next step in the development of laser technology for genetic investigation will be the use of laser beams as optical tweezers to extract the cut probes for further biological applications. In this case, laser cutting will be performed under aqueous conditions. As a consequence, the isolated chromosome sections will be floating and therefore susceptible to the dragging force of the optical tweezers. ${ }^{19,20}$ When a minimum size of the samples and touchless cutting is not required, alternative techniques such as AFM cutting should be used. However, the risk of contamination, especially in conjunction with the PCR technique, must be taken into account. DNA cutting under sterile conditions and amplification after nanoextraction of the dissected probe using special PCR techniques and performing in situ patterns will be described elsewhere.

\section{Acknowledgments}

The financial support of the Deutsche Forschungsgemeinschaft (He 1617/3-2) and Bayerische Forschungsstiftung is greatfully acknowledged.

\section{REFERENCES}

1. G. Senger, H. J. Luedecke, B. Horsthemke, and U. Claussen, "Microdissection of banded human chromosomes," Hum. Genet. 84, 507-511 (1990).

2. C. Lengauer, A. Ecklet, A. Weith, N. Endlich, N. Ponelies, P. Lichter, K. O. Greulich, and T. Cremer, "Painting of defined chromosomal regions by in situ suppression hybridisation of libraries from laser-microdissected chromosomes," Cytogenet. Cell. Genet. 56, 27-30 (1991).

3. E. Henderson, "Imaging and nanodissection of individual supercoiled plasmids by atomic force microscopy," Nucleic Acids Res. 20(3), 445-447 (1992).

4. D. M. Jondle, L. Ambrosio, J. Vesenka, and E. Henderson, "Imaging and manipulating chromosomes with the atomic force microscope," Chromosome Res. 3, 239-244 (1995).

5. H. J. Lüdecke, G. Senger, U. Claussen, and B. Horsthemke, "Cloning defined regions of human genome by microdissection of banded chromosomes and enzymatic amplification," Nature 338, 348-350 (1989).

6. B. G. de Groth and C. A. J. Putman, "High resolution imaging of chromosome-related structures by atomic force microscopy," J. Microsc. 168, 239-247 (1992).

7. W. M. Heckl and A. Engel, "Imaging nucleic acids with scanning probe microscopes," in Visualization of Nucleic Acids, Gerard Morel, Ed., CRC Press, Boca Raton, FL (1995).

8. P. Rasch, U. Wiedemann, J. Wienberg, and W. M. Heckl, "Analysis of banded human chromosomes and in situ hybridization patterns by scanning force microscopy," Proc. Natl. Acad. Sci. U.S.A. 90, 2509-2511 (1993).

9. N. van Hulst, M. Moers, and E. Borgonjen, "Applications of near field optical microscopy," in Photons and Local Probes, 
NATO ASI Series, O. Marti and R. Möller, Eds., Kluwer Academic, Dordrecht, The Netherlands.

10. W. Fritzsche, A. Schaper, and T. M. Jovin, "Probing chromatin structure with the scanning force microscope," Chromosoma 103, 231-236 (1994).

11. H. G. Hansma, J. Vesenka, C. Siegerist, G. Kelderman, H. Morrett, R. L. Sinsheimer, V. Elings, C. Bustamante, and P. K. Hansma, "Reproducible imaging and dissection of plasmid DNA under liquid with the atomic force microscope," Science 256, 1180-1184 (1992).

12. J. Conia and S. Völkel, "Optical manipulations of human gametes," BioTechniques 17(6), 1162-1165 (1994).

13. K. Schütze and A. Clement-Sengewald, "Catch and movecut and fuse," Nature 368, 667-669 (1994).

14. A. Clement-Sengewald, K. Schuetze, A. Askin, G. A. Palma, G. Kerlen, and G. Brem, "Fertilization of bovine oocytes induced Solely with combined laser microbeam and optical tweezers," J. Assisted Reproduc. Genet. 13(3), 259-265 (1996).

15. R. Stark, "Einsatz des Rasterkraftmikroskops als Nanoma- nipulator an DNA-Material und zur Entnahme von DNAProben," Diploma thesis Ludwig Maximilian University, München (1996).

16. P. Markiewicz and M. C. Goh, "Atomic force microscope tip deconvolution using calibration arrays," Rev. Sci. Instrum. 66(5), 3186-3190 (1995).

17. W. Fritzsche and E. Henderson, "Volume determination of human metaphase chromosomes by scanning force microscopy," Scanning Microscopy 10(1), 1-10 (1996).

18. E. H. K. Stelzer and S. Lindek, "Fundamental reduction of the observation volume in far-field light microscopy by detection orthagonal to the illumination axis: confocal thata microscopy," Opt. Commun. 111, 536-547 (1994).

19. S. Seeger, N. Leclerc, and E. H. K. Stelzer, "Photonic tweezers and laser nanoscalpel," Bioforum 17, 417-421 (1994).

20. K. Schütze, I. Becker, S. Thalhammer, R. Stark, W. M. Heckl, M. Boehm, and H. Poesl, "Cut out or poke in-the key to the world of single genes," Genetic Analysis: Biomolecular Engineering, 1996 (in press). 